\section{Reflexiones sobre la investigación esencial en Chile}

\author{
RODOLFO ARMAS MERINO ${ }^{1}$, ADRIÁN TORRES CANALES $^{2, a}$
}

\section{An analysis of essential health research in Chile}

Essential research studies of health problems affecting the majority of the population, aiming at actions that are feasible to be taken, efficiently and effectively implementing there and seeking solutions to unsolved problems. This is a complex process, which requires long lasting participation and coordinated interaction between different relevant sectors, namely the academic world, health policymakers and health-related industries. An analysis of essential health research in Chile is presented, considering factors such as shared efforts between the academic and health care sectors and the role of the Ministry of Health in research promotion. The following suggestions are made: 1) The Ministry of Health, along with universities, should stimulate, guide and monitor research activities that enrich and update the work on priority health issues; 2) To strengthen the capacity building of clinical or public health specialists by training them in applied research within medical centers, mainly teaching centers; 3) To assess the performance of National Fund for Health Research and Development (FONIS) and, if necessary, increase its resources to stimulate applied research in health; 4) To establish priorities for essential research, more specific than those proposed in 2010; 5) To reactivate the National Council for Health Research (CONIS) as an autonomous entity that coordinates applied research within the Ministry of Health.

(Rev Med Chile 2017; 145: 901-906)

Key words: Chile; Health Priorities; Health Services Research; Research.
'Departamento de Medicina Occidente, Universidad de Chile, Hospital San Juan de Dios. Santiago, Chile.

${ }^{2}$ Facultad de Ciencias Médicas Universidad de Santiago de Chile. aLicenciado en Filosofía.

Recibido el

aceptado el

Correspondencia a:

Rodolfo Armas Merino

Departamento de Medicina

Occidente, Universidad de Chile Hospital San Juan de Dios. Huérfanos 3255. Santiago, Chile. raarmas@mi.cl
E s un problema universal que los sistemas de salud pública no cuenten con la capacidad para resolver plenamente las necesidades sanitarias de la población. Las personas reclaman y sufren por las carencias en infraestructura, recursos humanos calificados y/o de presupuesto para enfrentar los gastos operacionales que demanda la medicina moderna. Nuestro país no es una excepción y aquí, como en todo el mundo, quienes más sufren y reclaman son las personas con menos recursos por ser las más dependientes de los sistemas públicos de salud. Para el bien de la humanidad, en el siglo XX hubo un progreso inimaginable de la medicina en la mantención y recuperación de la salud permitiendo que las per- sonas vivamos más y mejor, pero esto ha elevado los costos a niveles que sólo pueden solventar una minoría de las personas.

La investigación médica aporta conocimientos que permiten identificar mejor los problemas y enfrentarlos con más eficiencia. Por eso, se ha hecho necesario replantear las políticas de investigación orientándola a resolver necesidades concretas, fortalecer a las comunidades científicas y formar los recursos humanos necesarios.

Entre los investigadores del sector salud algunos tienen preferencia por los estudios próximos a las ciencias básicas y otros por temas relacionados con la práctica médica frecuente. La investigación básica no tiene en su perspectiva fines prácticos 
sino que apunta a contribuir al conocimiento general y al mejor entendimiento de la naturaleza y sus leyes y aparentemente se contrapone con la investigación que busca satisfacer necesidades sociales o de la práctica médica ${ }^{1}$. Quienes cultivan las investigaciones básicas y quienes se orientan a resolver los problemas sociales difieren en su formación, vocación, capacidades y ámbitos en que se desempeñan. No obstante, estas personas y estas actividades aparentemente disímiles, se complementan, pues las innovaciones que introduce la investigación aplicada habitualmente se basan en conocimientos aportados por la investigación básica.

Un tipo especial de investigación muy gravitante en el sector salud es la de la industria farmacéutica, la que invierte tanto en investigaciones básicas como aplicadas, pero con el interés en el retorno de la inversión.

Las investigaciones básicas y las aplicadas debieran mantener un fluido intercambio entre ellas y un apoyo equilibrado de parte de los Estados, las universidades, las empresas y otras instituciones que patrocinan y sostienen la actividad investigativa. Sin embargo, en 1994 la Organización Mundial de la Salud señaló que el 90\% de los esfuerzos y recursos destinados a investigación biomédica se destinaban a problemas que afectaban sólo al 10\% de las prioridades en salud pública a nivel global, destinando sólo 10\% de los recursos a solucionar problemas sanitarios que afectaban al $90 \%$ de la población mundial. Esto, conocido como la brecha 10/90, se explica porque el mayor aporte de la investigación ocurre en países ricos en los que no existen los problemas más frecuentes de salud como tuberculosis, malaria, desnutrición y otros propios de las sociedades pobres. Como reacción a la denuncia de esta brecha, se creó, entre otras instituciones, el Foro Mundial de Investigaciones Sanitarias para orientar iniciativas centradas en las afecciones que representan la mayor carga de morbilidad mundial, mejorar la asignación de los fondos de investigación y facilitar la cooperación entre los sectores público y privado. Es decir, se vio la urgencia de estimular la investigación en las necesidades, en la llamada "investigación esencial" o "por pertinencia", "por necesidad" o "aplicada" en contraposición con la investigación "por curiosidad" o "por el saber", que consiste en comprender los problemas que afectan a las mayorías, decidir las acciones que son factibles, ejecutar eficiente y efectivamente dichas acciones y buscar soluciones para problemas no resueltos ${ }^{2}$. Estas necesidades difieren de una a otra región dependiendo de los niveles socio-económicos y culturales. El estímulo a esta investigación requiere de formación de recursos humanos para enfrentar esta tarea ${ }^{3}$.

Cabe insistir en que la investigación esencial no se contrapone con la investigación básica que se genera por el interés propio de quien investiga. Al sistema universitario importa mucho la libertad y autonomía del investigador y acotarlas a intereses ajenos a éste es un camino seguro hacia la desvalorización de la investigación, de la ciencia, de los investigadores y de las universidades. Pero así como se entiende que en el ámbito industrial es razonable el desarrollo de la investigación en sintonía con los intereses de la correspondiente empresa, debe también entenderse que en el sector encargado de la salud pública se requiera de investigaciones aplicadas dirigidas a proporcionar mejor salud a las personas. Los países debieran apoyar e incentivar tanto las investigaciones básicas como las aplicadas, respetando la libertad de las primeras y acotando a las necesidades sociales las segundas; ambas son complementarias y los esfuerzos que en ellas invierte el Estado debieran tener un equilibrio razonable.

Consciente de esta situación, el año 2001 el Ministerio de Salud de Chile (MINSAL) creó el Consejo Nacional de Investigación en Salud $(\mathrm{CONIS})^{4}$ con el objetivo de "asesorar en forma permanente al ministro(a) de salud respecto a temas prioritarios que sean orientadores en materia de investigación científica y desarrollo tecnológico en salud". Constituían el Consejo representantes del MINSAL, de las sociedades médicas y de las facultades de Medicina y, abordó el tema a que se refiere este documento y otros de interés para el Ministerio como el de los ensayos clínicos que ocurren en establecimientos del Estado y sin regulación suficiente. Por su parte, el mismo año CONIS, conjuntamente con la Comisión Nacional de Investigación Científica y Tecnológica (CONICYT), crearon el Fondo Nacional de Investigación y Desarrollo en Salud (FONIS) para proporcionar recursos concursables para las investigaciones aplicadas, cuyos resultados contribuyan a mejorar o modificar, tanto las políticas públicas sanitarias, como su implementaciós, ${ }^{5,6}$. Este fondo se creó con escasos recursos pero se pensaba que crecería con 
el tiempo como ha ocurrido con todos los fondos de investigación.

En Chile, como en otros países latinoamericanos, la investigación médica ha estado radicada casi exclusivamente en el ámbito de las Universidades ${ }^{7}$. Pero, al crear el FONIS, el MINSAL dio una señal que ella no le es ajena por cuanto es necesaria para el desarrollo técnico y la incorporación de conocimientos y tecnologías al trabajo médico. Años después, en un documento sobre "Objetivos Sanitarios para el Año 2011-2020” se estableció la existencia del CONIS y del FONIS dentro de los objetivos ministeriales y se valoró la investigación en Salud". En ese documento se señala "Se deberá establecer una metodología de formulación de prioridades (priority setting) para la investigación en salud, que logre abarcar y concordar las diversas áreas temáticas que resulten relevantes para el país. Esta metodología establecerá prioridades de manera dinámica y será operacionalizada, entre otras instancias, a través de la plataforma del CONIS". No obstante ese texto, el CONIS ya no se citaba y no se citó nunca después.

En el curso del año 2008, CONIS se abocó a identificar las áreas que debieran ser prioritarias para la investigación sanitaria de interés para la salud pública. Para ello convocó a expertos provenientes de los sectores sanitarios, universitarios, fondos de investigación y de fomento de la producción, sociedades científicas médicas y empresas del área de la salud. El proceso enseñó que la priorización es un asunto complejo, que requiere de un abanico amplio de personas con intereses y sensibilidades diferentes ${ }^{9}$ y debe considerar factores muy diversos como son el bienestar de las personas, el gasto en salud, la morbimortalidad, sensibilidades ciudadanas, aspectos económicos y epidemiológicos y abarcar investigaciones de muchas áreas como son los estudios farmacológicos y de vacunas, los métodos diagnósticos, los comportamientos sociales, los factores de riesgo y temas de políticas, sistemas y gestión en salud. Después de varios meses de reuniones presenciales y consultas por vía electrónica se concordó que las prioridades para el MINSAL chileno de ese momento, debieran ser las señaladas en la Tabla $1^{10}$. Ese estudio ha sido muy considerado en intentos de priorización en el extranjero.

También enseñó ese estudio que, aunque se articulen los máximos niveles de salud, ciencia y tecnología, el liderazgo y dirección de la priorización debe radicarse en la autoridad sanitaria, a fin de que la diversidad de criterios e ideas que surjan así como los sesgos inevitables de las personas según la disciplina médica que cada cual cultiva, se supediten al objetivo de lograr un sistema eficiente con un impacto positivo en la salud pública. En otras palabras, asegurar la primacía de la pertinencia y el impacto social sobre la visibilidad política o académica de las propuestas.

La priorización necesita actualizarse Periódicamente, perfeccionándola y adecuándola a las

Tabla 1. Áreas Prioritarias para Investigación Esencial Propuesta por el Consejo Nacional de Salud del Ministerio de Salud de Chile en año 2010

1. Gestión y evaluación programas que contenía los siguientes temas:
a. Prevención primaria
b. Establecer prioridades en salud
c. Patología incluida en el programa de Garantías Explícitas en salud
d. Gestión y financiamiento de la atención de salud y
e. Participación ciudadana en el proceso de la atención médica

2. Patologías de alta prevalencia, incidencia, costos y/o impacto social que incluía:
a. Asuntos relacionados con salud mental
b. Patología pediátrica, de la adolescencia y del adulto mayor y
c. Problemas de salud con gran impacto social y

3. Patologías relacionadas con inequidades sociales, que incluía diversas condiciones sanitarias como desarrollo del niño, acceso a la salud, problema de salud mental, morbilidad y mortalidad general según características sociales, económicas, de género, de ruralidad o urbanismo de las personas 
realidades que van surgiendo como el desarrollo social, económico, cultural y epidemiológico de la sociedad y la incorporación de conocimientos médicos. En Chile por ejemplo, la priorización publicada el 2010 fue anterior a la instauración del Programa AUGE/GES que cambió radicalmente la atención de medicina curativa en el país.

Mirada retrospectivamente, esa priorización propuesta por CONIS ofreció temas muy amplios y pareciera que habría sido mejor proponer patologías más específicas.

Como ya señalamos, en abril de 2010 , en relación con un cambio de política ministerial se disolvió el CONIS el que nunca más se reactivó y tampoco ha habido esfuerzos en priorizar la investigación, aunque en los "Objetivos Sanitarios para el Año 2011-2020” se manifestó interés en contar con ella.

El FONIS es un fondo concursable altamente competitivo en el que logran financiamiento alrededor del $10 \%$ de quienes postulan y que ha jugado un importante papel en la investigación en salud en el país en los últimos 13 años. Financia proyectos de bajo costo y corta duración incentivando a participar a profesionales que se desempeñan directamente en el trabajo asistencial. Estas personas pueden hacer aportes efectivos para mejorar la toma de decisiones tanto en políticas sanitarias como en el trabajo clínico.

Como en todos los fondos concursables del país, en FONIS son las principales universidades las que más obtienen financiamiento para sus investigaciones, pero en éste, una proporción significativa $(15 \%)$ de las instituciones que postulan y logran financiamiento son universidades nuevas, hospitales, centros de salud, ONG y municipios $(\text { Tabla } 2)^{11}$. Los recursos aportados a FONIS han ido aumentando subiendo en de algo más de 300 millones en 2004 a sobre 1.000 millones en 2013. Es de desear que ese aporte mantenga su crecimiento porque es seguro que son de interés muchos proyectos presentados que no logran financiamiento.

Es sí preocupante que el año 2014 FONIS llamó a un concurso especial referente al programa Ministerial "Elige vivir sano". Sería esta una priorización temática impuesta sin un fundamento conocido; independiente de que sea éste un asunto digno de ser priorizado, hay que evitar actuar intuitivamente en esta materia

En agosto del año 2004 se dictó en Chile la Ley 19.966 que estableció un régimen de garantías en salud (GES), que alcanza a un cierto número de afecciones que son priorizadas garantizándoseles a quienes las sufren acceso oportuno a servicios de calidad. Inicialmente se dictó para veinte y ocho condiciones de salud seleccionadas por su alta prevalencia, impacto en términos de salud pública, calidad de vida para los pacientes y para sus grupos familiares. El programa ha ido ampliando las patologías que cubre y hoy día ellas son ochenta. Este fue un paso importante en el sistema de salud chileno, ya que a las afecciones elegidas se les da prioridad, cobertura económica y garantía de un manejo adecuado que debe ceñirse a plazos y normas preestablecidas. No obstante, en un estudio en que participamos ${ }^{12}$, comprobamos que pese a ser éstas patologías seleccionadas por su interés en la

Tabla 2. Número de proyectos aprobados en FONIS según instituciones (2004-2013)

\begin{tabular}{|lcccc|}
\hline Categoría de institución & \multicolumn{2}{c}{ Instituciones } & \multicolumn{2}{c|}{ Proyectos } \\
\hline Universidades* & $\mathbf{n}$ & $\mathbf{\%}$ & $\mathbf{n}$ & 82,6 \\
\hline Servicios de Salud & 24 & 38,1 & 239 & 4,8 \\
\hline ONGs & 11 & 17,5 & 14 & 4,5 \\
\hline Hospitales & 11 & 17,5 & 13 & 3,8 \\
Municipalidades & 8 & 12,7 & 11 & 1,7 \\
Otros & 5 & 7,9 & 5 & 2,4 \\
\hline Total & 4 & 6,3 & 289 & 100,0 \\
\hline
\end{tabular}

*Universidades: De Chile 91(38\%), Pontificia Católica de Chile 56 (23\%), de Concepción 14 (6 \%), de Valparaíso13 (5\%), otras veinte universidades 65 (27\%). 
salud pública, las 82 guías clínicas que regulaban hasta ese año su manejo, tenían como respaldo bibliográfico 6.604 referencias, de las cuales sólo $2,8 \%$ correspondían a estudios nacionales. Esto indicaría que los expertos que elaboraron esas guías prácticamente sólo confiaban en estudios extranjeros, evidenciando la carencia en Chile actual de investigación esencial en el área clínica o que los intereses de los mundos académico y asistencial caminan por carriles diferentes, paralelos y que no se encuentran ni se transfieren información (Tabla 3 ). Este divorcio entre investigadores y quienes diseñan políticas de salud fue ya detectado por Aranda y cols. de la Universidad de Chile, quienes informaron que en una encuesta a investigadores nacionales, $83 \%$ señalaron que los resultados de sus investigaciones no se incorporaron en las políticas de salud ${ }^{13}, 87 \%$ que no existen instancias formales de comunicación entre investigadores y formuladores de política y $54 \%$ señaló tener una "mala" y $6 \%$ una "muy mala" imagen de los formuladores de políticas sanitarias.

Es indispensable reducir la brecha entre el mundo de los conocimientos y el de la acción sanitaria, la que es bien conocida ${ }^{14}$, pero que entre nosotros es demasiado acentuada. Al MINSAL debiera llamarle la atención que los estudios financiados por FONIS -que él mismo creó para fortalecer la investigación aplicada nacional en salud- no aporten respaldo científico a las políticas de salud. Es posible que en algunas especialidades no exista actividad investigativa importante $y$, en tal caso, pareciera razonable incentivar su desarrollo. Esto no se corregirá de un día para otro y probablemente haya que reiniciar junto a las universidades acciones que este país ya implementó otrora, como es formar clínicos investigadores, crear centros especializados, estimular jornadas de trabajo de tiempo completo, incorporar en los servicio asistenciales a académicos calificados, formar en el extranjero a especialistas en áreas deficitarias, etc.
Del conjunto de antecedentes expuestos se puede concluir que la investigación para la salud es un proceso complejo, que requiere participación e interacción coordinada y mantenida en el tiempo de los diferentes sectores de relevancia para la innovación sanitaria, el mundo académico y determinadas industrias relacionadas con la salud y que debiera mantenerse con estabilidad ante los vaivenes políticos. El MINSAL y el sistema universitario trabajaron muy juntos en éste y otros problemas en tiempos del Servicio Nacional de Salud; después se produjo un alejamiento pero años más tarde el Ministerio creó el CONIS que fue una esperanza pero tuvo una efímera existencia.

Lo analizado en este documento nos permite concluir que:

- Sería deseable que el MINSAL, junto con las universidades estimulen, orienten y vigilen una actividad investigativa esencial que enriquezca y mantenga actualizada la labor sanitaria.

- Parece necesario evaluar el desempeño del FONIS para perfeccionarlo $y$, si fuese necesario y posible, aumentar sus recursos para estimular la investigación aplicada en salud. Evaluar por ejemplo cuántos proyectos han representado un aporte a la salud pública como impacto en términos de calidad y duración de vida, prevención de enfermedades, contención de costos, mayor eficiencia, etc.; qué instituciones de salud (públicas y privadas) se han beneficiado con este fondo (establecimientos públicos de atención primaria municipal, de nivel secundario o de mayor complejidad, universidades, etc.); qué temas de interés prioritario no se han abordado; cuántos proyectos han llegado a término y cuántos no lo han hecho; evaluar si es realista la duración preestablecida para cada proyecto; cuántos proyectos han dado lugar a una publicación y en qué órganos de difusión. No obstante hay que tener presente que el más valioso parámetro para medir el éxito de las investigaciones es su publicación en

Tabla 3. Origen de Estudios citados en Guías Clínicas de Programa Auge

\begin{tabular}{|c|c|c|c|}
\hline \multirow[t]{2}{*}{ Guías clínicas } & \multicolumn{3}{|c|}{ Referencias } \\
\hline & Total & Nacionales & Extranjeras \\
\hline 82 & $6.604(100 \%)$ & $185(2,8 \%)$ & $6.419(97,2 \%)$ \\
\hline
\end{tabular}

$8(0,12 \%)$ fueron proyectos de Fondos Concursables (Fondecyt 1, Fonis 5, Fondef 2). 
revistas de impacto internacional. No obstante, las investigaciones esenciales, independientes de su calidad, frecuentemente no gozan de la predilección de los comités editoriales precisamente por su carácter regional y no universal. - Dado que la escasez de personas interesadas y con tiempo protegido para investigar es probablemente uno de los principales escollos para esta actividad ${ }^{15}$, cabría explorar la formación de especialistas clínicos o en salud pública con adiestramiento en investigación dentro de las unidades docente-asistenciales;

- Convendría establecer un listado de afecciones prioritarias para la investigación, más específico que el del año 2010, elaborado a través de un programa multidisciplinario con participación de profesionales que cultiven diferentes áreas de la medicina, epidemiólogos, representantes ministeriales y universitarios.

- Parece necesario reactivar el CONIS para que coordine con autonomía la investigación aplicada al interior del MINSAL.

En fin, hay una situación preocupante en la investigación aplicada en el país que requiere de una acción mancomunada de los sectores académicos y asistenciales.

\section{Referencias}

1. Pellegrini A. Ciencia en pro de la salud, Notas sobre la organización de la actividad científica para el desarrollo de la salud en América Latina y el Caribe. Washington DC: OPS 2000.

2. Commission on Health Research for Development. Health Research. Essential link to equity in development. Oxford: Oxford University Press, 1990.

3. Pinto Bustamante BJ, Mojica MC, Gulfo Díaz R, Endo Pascuas JH, Idárraga J. Bioética y la brecha10/90: fallos, desafíos y oportunidades. Revista Red bioética/UNESCO
2014; 5 (2): 81-93.

4. Ministerio de Salud, Decreto No 145 de 2001.

5. Valdivieso V. El futuro de la investigación en salud. Visión de las universidades. Boletín Academia Chilena de Medicina 2001; 38 (1): 89-93.

6. Armas Merino R. Hacia un fondo nacional de investigaciones aplicadas en salud. Boletín Academia Chilena de Medicina 2001; 38 (1): 95-9.

7. Valdés G, Armas MR, Reyes BH. Principales características de la investigación biomédica actual, en Chile. Rev Med Chile 2012; 140 (4): 484-92.

8. Ministerio de Salud. Estrategia Nacional de Salud Para el cumplimiento de los Objetivos Sanitarios de la Década 2011-2020. Objetivo $7^{\circ}$.

9. Agudelo CA, De la Hoz F, Mojica MJ, Eslava JC, Robledo R, Cifuentes $\mathrm{P}$, et al. Prioridades de Investigación en Salud en Colombia: Perspectiva de los Investigadores. Rev Salud pública (Colombia) 2009; 11 (2): 301-9.

10. Armas Merino R, Torres CA, Arriagada CJ, Muñoz PF, Salinas RR, Crocco AP. Proceso para priorizar las líneas de investigación esencial de interés para el Ministerio de Salud de Chile. Rev Med Chile 2010; 138 (4): 401-5.

11. Luengo MX. Chile: La buena salud de la investigación en salud. (Editorial) Rev Chil Salud Pública 2012; 16 (3): 208-9.

12. Núñez FP, Torres CA, Armas MR. ¿Se sustentan las Guías GES en trabajos científicos financiados por los fondos de CONICYT? Rev Med Chile 2014; 142 (12): 1540-6.

13. Aranda W, Torres GA, Tapia E, Solimano G, Alarcón A. Percepción de Investigadores/Investigadoras y Utilización de resultados en Políticas de Salud en Chile. En Memorias de III Congreso Latinoamericano y del Caribe de Salud Global. San José de Costa Rica, 2014.

14. Haines A, Kuruvilla S, Borchert M. Bridging the implementation gap between knowledge and action for health. Bull World Health Organ 2004; 82 (10): 72431.

15. Valdés SG. Una mirada a la investigación biomédica actual en Chile. Boletín de la Academia Chilena de Medicina 2011; 48 (1): 67-89. 